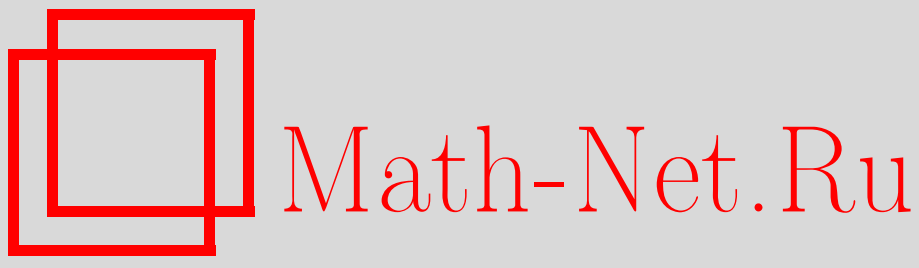

М. Ш. Шабозов, М. С. Саидусайнов, Приближение функций комплексного переменного суммами Фурье по ортогональным системам в $L_{2}$, Изв. вузов. Матем., 2020, номер 6, 65-72

DOI: https://doi.org/10.26907/0021-3446-2020-6-65-72

Использование Общероссийского математического портала Math-Net.Ru подразумевает, что вы прочитали и согласны с пользовательским соглашением

http://www . mathnet.ru/rus/agreement

Параметры загрузки:

IP: 54.80 .97 .219

26 апреля 2023 г., 14:55:32

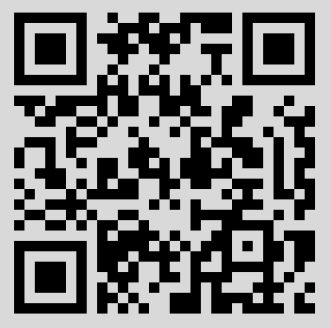




\section{М.Ш.ШАБОЗОВ, М.С.САИДУСАЙНОВ}

\section{ПРИБЛИЖЕНИЕ ФУНКЦИЙ КОМПЛЕКСНОГО ПЕРЕМЕННОГО СУММАМИ ФУРЬЕ ПО ОРТОГОНАЛЬНЫМ СИСТЕМАМ В $L_{2}$}

Аннотация. Получены точные неравенства типа Джексона-Стечкина между величиною наилучшего приближения $E_{n-s-1}\left(f^{(s)}\right)(s=\overline{0, r}, r \in \mathbb{N})$ последовательности производных $f^{(s)}(s=$ $\overline{0, r}, r \in \mathbb{N})$ аналитических в круге $U:=\{z:|z|<1\}$ функций $f \in L_{2}(U)$ как для специального модуля непрерывности $m$-го порядка $\Omega_{m}$, удовлетворяющего условию

$$
\Omega_{m}\left(f^{(r)}, t\right)_{2} \leq \Phi(t), 0<t<1,
$$

где $\Phi-$ заданная мажоранта, так и для $\mathscr{K}$-функционала Петре, удовлетворяющего ограничению

$$
\mathscr{K}_{m}\left(f^{(r)}, t^{m}\right) \leq \Phi\left(t^{m}\right), 0<t<1 .
$$

Ключевые слова: обобщенный модуль непрерывности, оператор обобщенного сдвига, ортонормированная система функций, неравенство Джексона-Стечкина, $\mathscr{K}$-функционал.

\section{УДК: 517.5}

DOI: 10.26907/0021-3446-2020-6-65-72

В работе рассматривается задача среднеквадратичного полиномиального приближения функций комплексного переменного, регулярных в некоторой односвязной области $\mathcal{D} \subset \mathbb{C}$, суммами Фурье по ортогональной системе в пространстве $L_{2}:=L_{2}(\mathcal{D})$ с конечной нормой

$$
\|f\|_{2}:=\|f\|_{L_{2}(\mathcal{D})}=\left(\frac{1}{\pi} \iint_{(\mathcal{D})}|f(z)|^{2} d \sigma\right)^{1 / 2},
$$

где интеграл понимается в смысле Лебега, $d \sigma-$ элемент площади.

Отметим, что различные аспекты теории ортогональных по области регулярных функций и аппроксимации в среднем функций $f \in L_{2}(\mathcal{D})$ суммами Фурье рассмотрены в монографии ([1], гл.III, с. 196-278). В [2], [3] изучена задача отыскания точной константы в неравенстве типа Джексона-Стечкина между величиной наилучшего среднеквадратичного приближения функций $f \in L_{2}(\mathcal{D})$ и обобщенным модулем непрерывности высшего порядка, а в [4] приведено некоторое уточнение неравенства Джексона-Стечкина из [2]. В случае, когда $\mathcal{D}:=U=\{z \in \mathbb{C}:|z|<1\}$, в [5] получены точные оценки скорости сходимости рядов Фурье по ортогональной в круге $U$ системе $\left\{z^{k}\right\}_{k=0}^{\infty}$ на классах функций, задаваемых обобщенным модулем непрерывности $m$-го порядка, и вычислены значения ряда $n$-поперечников на указанных классах функций. Здесь мы продолжим исследования в этом направлении и попутно обобщим некоторые результаты из [5].

Поступила в редакцию 25.06.2019, после доработки 31.07.2019. Принята к публикации 25.09.2019. 
Далее рассмотрим случай, когда $\mathcal{D}$ есть круг $U:=\{z \in \mathbb{C}:|z|<1\}$. Очевидно, что в этом случае система функций $\varphi_{k}(z)=z^{k}, k \in \mathbb{Z}_{+}$, является ортогональной в круге $U$ :

$$
\frac{1}{\pi} \iint_{(U)} \varphi_{k}(z) \overline{\varphi_{l}(z)} d \sigma=\frac{1}{\pi} \int_{0}^{1} \int_{0}^{2 \pi} r^{k+l+1} e^{i(k-l) t} d r d t=0, k \neq l .
$$

Но эта система не является ортонормированной, поскольку

$$
\frac{1}{\pi} \iint_{(U)}\left|\varphi_{k}(z)\right|^{2} d \sigma=\frac{1}{\pi} \int_{0}^{1} \int_{0}^{2 \pi} r^{2 k+1} d r d t=\frac{1}{k+1} .
$$

Следовательно, система функций $\varphi_{k}^{*}(z)=\sqrt{k+1} z^{k}, k \in \mathbb{Z}_{+}$, является ортонормированной системой. Через $\mathcal{A}(U)$ обозначим множество аналитических в $U$ функций $f$. Ряд Маклорена функции $f \in \mathcal{A}(U)$ имеет вид

$$
f(z)=\sum_{k=0}^{\infty} c_{k}(f) z^{k}
$$

где $c_{k}(f)$ - коэффициенты Маклорена функции $f$. При этом

$$
\|f\|_{2}^{2}=\sum_{k=0}^{\infty} \frac{\left|c_{k}(f)\right|^{2}}{k+1}, \quad E_{n-1}^{2}(f)_{2}=\left\|f-S_{n-1}(f)\right\|^{2}=\sum_{k=n}^{\infty} \frac{\left|c_{k}(f)\right|^{2}}{k+1} .
$$

В монографии ([1], с. 209) доказано, что ряд Фурье функции $f$ по ортонормированной системе $\varphi_{k}^{*}(z)=\sqrt{k+1} z^{k}, k \in \mathbb{Z}_{+}$, совпадает с рядом (1) функций $f \in \mathcal{A}(U)$, т. е.

$$
f(z)=\sum_{k=0}^{\infty} a_{k}(f) \varphi_{k}^{*}(z)=\sum_{k=0}^{\infty} c_{k}(f) z^{k}
$$

а потому ряд (1) можно сколько угодно раз почленно продифференцировать и по теореме Вейерштрасса ([6], с. 107) для произвольного $r \in \mathbb{N}$ будем иметь

$$
f^{(r)}(z)=\sum_{k=r}^{\infty} c_{k}(f) k(k-1) \cdots(k-r+1) z^{k-r}:=\sum_{k=r}^{\infty} \alpha_{k, r} c_{k}(f) z^{k-r},
$$

где

$$
\alpha_{k, r}:=k(k-1) \cdots(k-r+1), k \in \mathbb{N}, r \in \mathbb{Z}_{+}, k \geq r .
$$

При этом легко подсчитать, что при любом $0 \leq s \leq r, s, r \in \mathbb{Z}_{+}$, имеет место равенство

$$
E_{n-s-1}^{2}\left(f^{(s)}\right)_{2}=\left\|f^{(s)}-S_{n-s-1}\left(f^{(s)}\right)\right\|_{2}^{2}=\sum_{k=n}^{\infty} \alpha_{k, s}^{2} \frac{\left|c_{k}(f)\right|^{2}}{k-s+1} .
$$

Обозначим через $L_{2}^{(r)}:=L_{2}^{(r)}(U)\left(r \in \mathbb{Z}_{+}, L_{2}^{(0)}:=L_{2}(U)\right)$ класс функций $f \in L_{2}$, у которых $f^{(r)} \in L_{2}\left(r \in \mathbb{Z}_{+}, f^{(0)} \equiv f\right)$.

В [5] при любых $n, m \in \mathbb{N}, r, s \in \mathbb{Z}_{+}$и в предположении $n>r \geq s$ доказаны равенства

$$
\begin{gathered}
\sup _{f \in L_{2}^{(r)}} \frac{E_{n-s-1}\left(f^{(s)}\right)_{2}}{E_{n-r-1}\left(f^{(r)}\right)_{2}}=\sqrt{\frac{n-r+1}{n-s+1}} \frac{\alpha_{n, s}}{\alpha_{n, r}}, \\
\Omega_{m}^{2}\left(f^{(r)} ; t\right)_{2}=\sum_{k=r}^{\infty}\left[1-(1-t)^{k-r}\right]^{2 m} \alpha_{k, r}^{2} \frac{\left|c_{k}(f)\right|^{2}}{k-r+1}, 0<t<1 .
\end{gathered}
$$

Имеет место следующая 
Теорема 1. При любых $m, n \in \mathbb{N}, r, s \in \mathbb{Z}_{+}, n>r \geq s u t \in(0,1)$ справедливо равенство

$$
\sup _{f \in L_{2}^{(r)}} \frac{\sqrt{(n-s+1) /(n-r+1)}\left(\alpha_{n, r} / \alpha_{n, s}\right) E_{n-s-1}\left(f^{(s)}\right)_{2}}{\Omega_{m}\left(f^{(r)} ; t\right)_{2}}=\frac{1}{\left[1-(1-t)^{n-r}\right]^{m}} .
$$

В частности, из (9) при любых $m, n \in \mathbb{N}, r, s \in \mathbb{Z}_{+}, n>r \geq s$ вытекает соотношение

$$
\sup _{n \in \mathbb{N}} \sup _{f \in L_{2}^{(r)}} \frac{\sqrt{(n-s+1) /(n-r+1)}\left(\alpha_{n, r} / \alpha_{n, s}\right) E_{n-s-1}\left(f^{(s)}\right)_{2}}{\Omega_{m}\left(f^{(r)} ; 1 /(n-r)\right)_{2}}=\left(1-e^{-1}\right)^{-m} .
$$

Доказательство. Пользуясь равенством (8) и учитывая соотношение (6), при любых $m, n \in$ $\mathbb{N}, r \in \mathbb{Z}_{+}, n>r$ и $t \in(0,1)$ для произвольной функции $f \in L_{2}^{(r)}$ будем иметь

$$
\begin{gathered}
\Omega_{m}^{2}\left(f^{(r)} ; t\right)_{2}=\sum_{k=r}^{\infty}\left[1-(1-t)^{k-r}\right]^{2 m} \alpha_{k, r}^{2} \frac{\left|c_{k}(f)\right|^{2}}{k-r+1} \geq \sum_{k=n}^{\infty}\left[1-(1-t)^{k-r}\right]^{2 m} \alpha_{k, r}^{2} \frac{\left|c_{k}(f)\right|^{2}}{k-r+1} \geq \\
\geq\left[1-(1-t)^{n-r}\right]^{2 m} E_{n-r-1}^{2}\left(f^{(r)}\right)_{2} .
\end{gathered}
$$

Согласно (7) запишем (11) в виде

$$
\Omega_{m}\left(f^{(r)} ; t\right)_{2} \geq\left[1-(1-t)^{n-r}\right]^{m} \frac{\alpha_{n, r}}{\alpha_{n, s}} \sqrt{\frac{n-s+1}{n-r+1}} E_{n-s-1}\left(f^{(s)}\right)_{2},
$$

откуда и вытекает оценка сверху величины, стоящей в левой части (9).

С целью получения аналогичной оценки снизу вводим функцию $f_{0}(z)=z^{n} \in L_{2}^{(r)}$. Для этой функции, как следует из равенств (6) и (8), имеем

$$
E_{n-s-1}\left(f_{0}^{(s)}\right)_{2}=\frac{\alpha_{n, s}}{\sqrt{n-s+1}}, \Omega_{m}\left(f_{0}^{(r)} ; t\right)_{2}=\left[1-(1-t)^{n-r}\right]^{m} \frac{\alpha_{n, r}}{\sqrt{n-r+1}} .
$$

Воспользуясь равенствами (13), запишем оценку снизу указанной величины

$$
\begin{gathered}
\sup _{f \in L_{2}^{(r)}} \frac{\sqrt{(n-s+1) /(n-r+1)}\left(\alpha_{n, r} / \alpha_{n, s}\right) E_{n-s-1}\left(f^{(s)}\right)_{2}}{\Omega_{m}\left(f^{(r)} ; t\right)_{2}} \geq \\
\geq \frac{\sqrt{(n-s+1) /(n-r+1)}\left(\alpha_{n, r} / \alpha_{n, s}\right) E_{n-s-1}\left(f_{0}^{(s)}\right)_{2}}{\Omega_{m}\left(f_{0}^{(r)} ; t\right)_{2}}= \\
=\frac{\sqrt{(n-s+1) /(n-r+1)}\left(\alpha_{n, r} / \alpha_{n, s}\right)\left(\alpha_{n, s} / \sqrt{n-s+1}\right)}{\left[1-(1-t)^{n-r}\right]^{m}\left(\alpha_{n, r} / \sqrt{n-r+1}\right)}=\frac{1}{\left[1-(1-t)^{n-r}\right]^{m}} .
\end{gathered}
$$

Из неравенства (12) и (14) следует требуемое равенство (9). Полагая в (9) $t=1 /(n-r)$ и переходя к верхней грани по всем $n \in \mathbb{N}$ (при фиксированном $r \in \mathbb{Z}_{+}$), получим соотношение (10), чем и завершаем доказательство теоремы 1 . Отметим, что при $s=0$ теорема 1 ранее доказана в [5].

Далее приводим точные неравенства, связывающие величину наилучшего приближения $E_{n-1}\left(f^{(s)}\right)_{2}(s=\overline{0, r})$ функций $f \in L_{2}^{(r)}$ посредством $\mathscr{K}$-функционала Петре [7]-[9]. Определим $\mathscr{K}$-функционал, построенный по пространствам $L_{2}$ и $L_{2}^{(m)}, m \in \mathbb{N}$ :

$$
\mathscr{K}_{m}\left(f ; t^{m}\right):=\mathscr{K}\left(f ; t^{m} ; L_{2}, L_{2}^{(m)}\right)=\inf \left\{\|f-g\|_{2}+t^{m}\left\|g^{(m)}\right\|_{2}: g \in L_{2}^{(m)}\right\} .
$$


Представляет интерес точно вычислить экстремальную характеристику величины, подобной левой части равенства (9), но содержащей вместо модуля непрерывности $\Omega_{m} m$-го порядка $\mathscr{K}$-функционал (15).

Теорема 2. Пусть $m, n \in \mathbb{N}, r, s \in \mathbb{Z}_{+}(r \geq s)$ - произвольные числа такие, что $n \geq r+m$. Тогда справедливо равенство

$$
\sup _{\substack{f \in L_{2}^{(r)} \\ f \in \mathcal{P}_{r}}} \frac{\sqrt{(n-s+1) /(n-r+1)}\left(\alpha_{n, r} / \alpha_{n, s}\right) E_{n-s-1}\left(f^{(s)}\right)_{2}}{\mathscr{K}_{m}\left(f^{(r)}, \sqrt{\frac{n-r-m+1}{n-r+1}} \frac{1}{\alpha_{n-r, m}}\right)}=1 .
$$

Доказательство. Используя равенство (7), для произвольной функции $f \in L_{2}^{(r)}$ запишем

$$
E_{n-s-1}\left(f^{(s)}\right)_{2} \leq \sqrt{\frac{n-r+1}{n-s+1}} \frac{\alpha_{n, s}}{\alpha_{n, r}} E_{n-r-1}\left(f^{(r)}\right)_{2} \leq \sqrt{\frac{n-r+1}{n-s+1}} \frac{\alpha_{n, s}}{\alpha_{n, r}}\left\|f^{(r)}-S_{n-r-1}(g)\right\|_{2},
$$

где $S_{n-r-1}(g)$ - частная сумма $(n-r)$-го порядка ряда Фурье произвольной функции $g \in$ $L_{2}^{(m)}$. В силу неравенства $(2.12)$ из [5] для $g \in L_{2}^{(m)}$ получаем

$$
\begin{gathered}
\left\|g-S_{n-r-1}(g)\right\|=E_{n-r-1}(g)_{2} \leq \sqrt{\frac{n-r-m+1}{n-r+1}} \frac{1}{\alpha_{n-r, m}} E_{n-r-m-1}\left(g^{(m)}\right)_{2} \leq \\
\leq \sqrt{\frac{n-r-m+1}{n-r+1}} \frac{1}{\alpha_{n-r, m}}\left\|g^{(m)}\right\|_{2} .
\end{gathered}
$$

Из неравенств (17) и (18) вытекает

$$
\begin{aligned}
& E_{n-s-1}\left(f^{(s)}\right)_{2} \leq \sqrt{\frac{n-r+1}{n-s+1}} \frac{\alpha_{n, s}}{\alpha_{n, r}}\left\{\left\|f^{(r)}-g\right\|_{2}+\left\|g-S_{n-r-1}(g)\right\|_{2}\right\} \leq \\
& \leq \sqrt{\frac{n-r+1}{n-s+1}} \frac{\alpha_{n, s}}{\alpha_{n, r}}\left\{\left\|f^{(r)}-g\right\|_{2}+\sqrt{\frac{n-r-m+1}{n-r+1}} \frac{1}{\alpha_{n-r, m}}\left\|g^{(m)}\right\|_{2}\right\} .
\end{aligned}
$$

Левая часть неравенства (19) не зависит от функции $g \in L_{2}^{(m)}$, а потому, минимизируя его правую часть по $g \in L_{2}^{(m)}$ с учетом определения $\mathscr{K}$-функционала (15), получаем

$$
E_{n-s-1}(f)_{2} \leq \sqrt{\frac{n-r+1}{n-s+1}} \frac{\alpha_{n, s}}{\alpha_{n, r}} \mathscr{K}_{m}\left(f^{(r)}, \sqrt{\frac{n-r-m+1}{n-r+1}} \frac{1}{\alpha_{n-r, m}}\right) .
$$

Отсюда следует оценка сверху величины, стоящей в левой части равенства (16):

$$
\sup _{\substack{f \in L_{2}^{(r)} \\ f \in \mathcal{P}_{r}}} \frac{\sqrt{(n-s+1) /(n-r+1)}\left(\alpha_{n, r} / \alpha_{n, s}\right) E_{n-1}(f)_{2}}{\mathscr{K}_{m}\left(f^{(r)}, \sqrt{\frac{n-r-m+1}{n-r+1}} \frac{1}{\alpha_{n-r, m}}\right)_{2}} \leq 1 .
$$

С целью получения оценки снизу той же величины, воспользуемся тем, что для произвольной $p_{n} \in \mathscr{P}_{n}$ имеет место неравенство (см., например, [10])

$$
\mathscr{K}_{m}\left(p_{n}, t^{m}\right) \leq \min \left\{\left\|p_{n}\right\|_{2}, t^{m}\left\|p_{n}^{(m)}\right\|_{2}\right\} .
$$

Рассмотрим функцию $f_{0}(z)=z^{n}$. Так как для этой функции

$$
f_{0}^{(r+m)}=n(n-1) \cdots(n-r+1)(n-r) \cdots(n-r-m+1) z^{n-r-m}=\alpha_{n, r} \alpha_{n-r, m} z^{n-(r+m)},
$$


то в силу неравенства (21) будем иметь

$$
\begin{gathered}
\mathscr{K}_{m}\left(f_{0}^{(r)} ; \sqrt{\frac{n-r-m+1}{n-r+1}} \frac{1}{\alpha_{n-r, m}}\right) \leq \sqrt{\frac{n-r-m+1}{n-r+1}} \frac{1}{\alpha_{n-r, m}}\left\|f_{0}^{(r+m)}\right\|_{2}= \\
=\sqrt{\frac{n-r-m+1}{n-r+1}} \frac{1}{\alpha_{n-r, m}} \frac{\alpha_{n, r} \alpha_{n-r, m}}{\sqrt{n-r-m+1}}=\frac{\alpha_{n, r}}{\sqrt{n-r+1}} .
\end{gathered}
$$

Заметим также, что

$$
E_{n-s-1}\left(f_{0}^{(s)}\right)_{2}=\frac{\alpha_{n, s}}{\sqrt{n-s+1}} .
$$

Пользуясь соотношениями (22) и (23), имеем

$$
\begin{gathered}
\sup _{\substack{f \in L_{2}^{(r)} \\
f \in \mathcal{P}_{r}}} \frac{\sqrt{(n-s+1) /(n-r+1)}\left(\alpha_{n, r} / \alpha_{n, s}\right) E_{n-s-1}\left(f^{(s)}\right)_{2}}{\mathscr{K}_{m}\left(f_{0}^{(r)}, \sqrt{\frac{n-r-m+1}{n-r+1}} \frac{1}{\alpha_{n-r, m}}\right)} \geq \\
\geq \frac{\sqrt{(n-s+1) /(n-r+1)}\left(\alpha_{n, r} / \alpha_{n, s}\right) E_{n-1}\left(f_{0}\right)_{2}}{\mathscr{K}_{m}\left(f_{0}^{(r)}, \sqrt{\frac{n-r-m+1}{n-r+1}} \frac{1}{\alpha_{n-r, m}}\right)}=\frac{\alpha_{n, r} / \sqrt{n-r+1}}{\mathscr{K}\left(f_{0}^{(r)}, \sqrt{\frac{n-r-m+1}{n-r+1}} \frac{1}{\alpha_{n-r, m}}\right)} \geq 1 .
\end{gathered}
$$

Требуемое равенство (16) получаем из сравнения оценки сверху (20) с оценкой снизу (24).

Заметим, что из результата теоремы 1 (равенство (9)) и теоремы 2 (равенство (16)) после некоторых простых вычислений вытекает асимптотическое равенство

$$
\mathscr{K}_{m}\left(f^{(r)}, t^{m}\right) \sim \Omega_{m}\left(f^{(r)}, t\right), \quad 0<t<1 .
$$

Неубывающая на $\mathbb{R}_{+}$функция $\Phi$ называется $k$-мажорантой ([11], с. 25$)$, если функция $t^{-k} \Phi(t)$ не возрастает на $\mathbb{R}_{+}, \Phi(0)=0$ и $\Phi(t) \rightarrow 0$ при $t \rightarrow 0$. При $k=1$ функцию $\Phi$ называют просто мажорантой. Через $W_{2}^{(r)}\left(\Omega_{m}, \Phi\right)\left(r \in \mathbb{Z}_{+}, m \in \mathbb{N}\right)$ обозначим класс функций, состоящих из элементов $f \in L_{2}^{(r)}$, у которых производные $f^{(r)}$ удовлетворяют условию

$$
\Omega_{m}\left(f^{(r)}, t\right) \leq \Phi(t), \quad 0<t<1,
$$

а через $W_{2}^{(r)}\left(\mathscr{K}_{m}, \Phi\right)\left(r \in \mathbb{Z}_{+}, m \in \mathbb{N}\right)$ обозначим аналогичный класс функций, $f \in L_{2}^{(r)}$, для которых

$$
\mathscr{K}_{m}\left(f^{(r)}, t^{m}\right) \leq \Phi\left(t^{m}\right), \quad 0<t<1 .
$$

Далее решаем следующую задачу: пусть $\mathfrak{M}^{(r)}-$ некоторый подкласс функций класса $L_{2}^{(r)}$. Требуется найти величину

$$
E_{n-s-1}\left(\mathfrak{M}^{(r)}\right)_{2}:=\sup \left\{E_{n-s-1}\left(f^{(s)}\right)_{2}: f \in \mathfrak{M}^{(r)}\right\} .
$$

Приводим решение задачи $(25)$ для классов функций $W_{2}^{(r)}\left(\Omega_{m}, \Phi\right)$ и $W_{2}^{(r)}\left(\mathscr{K}_{m}, \Phi\right)$.

Теорема 3. Пусть $\Phi-$ мажоранта, определяющая класс функций $W_{2}^{(r)}\left(\Omega_{m}, \Phi\right)$, где $m, r \in$ $\mathbb{N}, r \geq 2$. Тогда для любого натурального $n \geq m+r, t \in(0,1)$ и произвольного $s(0 \leq s \leq r)$ справедливо равенство

$$
E_{n-s-1}\left(W_{2}^{(r)}\left(\Omega_{m}, \Phi\right)\right)_{2}=\sqrt{\frac{n-r+1}{n-s+1}} \frac{\alpha_{n, s}}{\alpha_{n, r}} \frac{\Phi(t)}{\left[1-(1-t)^{n-r}\right]^{m}} .
$$


Доказательство. Действительно, из равенства (9) для произвольной функции $f \in L_{2}^{(r)}$ получаем

$$
E_{n-s-1}\left(f^{(s)}\right)_{2} \leq \sqrt{\frac{n-r+1}{n-s+1}} \frac{\alpha_{n, s}}{\alpha_{n, r}} \frac{\Omega_{m}\left(f^{(r)}, t\right)_{2}}{\left[1-(1-t)^{n-r}\right]^{m}},
$$

и если теперь предполагать, что $f \in L_{2}^{(r)} \cap W_{2}^{(r)}\left(\Omega_{m}, \Phi\right)$, то из $(27)$ сразу следует оценка сверху величины, расположенной в левой части равенства (26):

$$
E_{n-s-1}\left(W_{2}^{(r)}\left(\Omega_{m}, \Phi\right)\right)_{2} \leq \sqrt{\frac{n-r+1}{n-s+1}} \frac{\alpha_{n, s}}{\alpha_{n, r}} \frac{\Phi(t)}{\left[1-(1-t)^{n-r}\right]^{m}} .
$$

Введем функцию

$$
g_{0}(z)=\frac{\sqrt{n-r+1}}{\alpha_{n, r}} \frac{\Phi(t) z^{n}}{\left[1-(1-t)^{n-r}\right]^{m}}, \quad 0<t<1 .
$$

Для этой функции легко проверить, что

$$
\begin{gathered}
g_{0}^{(s)}(z)=\frac{\sqrt{n-r+1}}{\alpha_{n, r}} \alpha_{n, s} \frac{\Phi(t) z^{n-s}}{\left[1-(1-t)^{n-r}\right]^{m}}, \\
E_{n-s-1}\left(g_{0}^{(s)}\right)_{2}=\sqrt{\frac{n-r+1}{n-s+1}} \frac{\alpha_{n, s}}{\alpha_{n, r}} \frac{\Phi(t)}{\left[1-(1-t)^{n-r}\right]^{m}}, \\
\Omega_{m}\left(g_{0}^{(s)}, t\right)_{2}=\Phi(t), \quad 0<t<1 .
\end{gathered}
$$

Последнее равенство означает, что $g_{0}(z) \in W_{2}^{(r)}\left(\Omega_{m}, \Phi\right)$. Учитывая равенство (29), запишем оценку снизу искомой величины

$$
E_{n-s-1}\left(W_{2}^{(r)}\left(\Omega_{m}, \Phi\right)\right)_{2} \geq E_{n-s-1}\left(g_{0}^{(s)}\right)_{2}=\sqrt{\frac{n-r+1}{n-s+1}} \frac{\alpha_{n, s}}{\alpha_{n, r}} \frac{\Phi(t)}{\left[1-(1-t)^{n-r}\right]^{m}} .
$$

Из сравнения неравенств (28) и (30) следует равенство (26), чем и завершаем доказательство теоремы 3.

Заметим, что при $t=1 /(n-r), n>r, n, r \in \mathbb{N}$ из (26) следует асимптотическое равенство

$$
E_{n-s-1}\left(W_{2}^{(r)}\left(\Omega_{m}, \Phi\right)\right)_{2} \asymp \sqrt{\frac{n-r+1}{n-s+1}} \frac{\alpha_{n, s}}{\alpha_{n, r}} \Phi\left(\frac{1}{n-r}\right)\left(1-\frac{1}{e}\right)^{-m} .
$$

Теорема 4. Пусть $\Phi-$ мажсоранта, определяющая класс функиий $W_{2}^{(r)}\left(\mathscr{K}_{m}, \Phi\right)$, где $m, r \in$ $\mathbb{N}, r \geq 2$. Тогда для любого натурального $n \geq m+r$ и произвольного $s(1 \leq s \leq r-1)$ справедливо равенство

$$
E_{n-s-1}\left(W_{2}^{(r)}\left(\mathscr{K}_{m}, \Phi\right)\right)_{2}=\sqrt{\frac{n-r+1}{n-s+1}} \frac{\alpha_{n, s}}{\alpha_{n, r}} \Phi\left(\sqrt{\frac{n-r-m+1}{n-r+1}} \frac{1}{\alpha_{n-r, m}}\right) .
$$

Доказательство. Действительно, оценка сверху величины, расположенной в левой части $(31)$, следует из равенства $(16)$, в силу которой для произвольной функции $f \in W_{2}^{(r)}\left(\mathscr{K}_{m}, \Phi\right)$ получаем

$$
E_{n-s-1}\left(f^{(s)}\right)_{2} \leq \sqrt{\frac{n-r+1}{n-s+1}} \frac{\alpha_{n, s}}{\alpha_{n, r}} \mathscr{K}_{m}\left(f^{(r)}, \sqrt{\frac{n-r-m+1}{n-r+1}} \frac{1}{\alpha_{n-r, m}}\right)_{2} \leq
$$




$$
\leq \sqrt{\frac{n-r+1}{n-s+1}} \frac{\alpha_{n, s}}{\alpha_{n, r}} \Phi\left(\sqrt{\frac{n-r-m+1}{n-r+1}} \frac{1}{\alpha_{n-r, m}}\right) .
$$

В [10] доказано, что функция

$$
g_{0}(z)=\frac{\sqrt{n-r+1}}{\alpha_{n, r}} \Phi\left(\sqrt{\frac{n-r-m+1}{n-r+1}} \frac{1}{\alpha_{n-r, m}}\right) z^{n}
$$

принадлежит классу $W_{2}^{(r)}\left(\mathscr{K}_{m}, \Phi\right)$, и так как для этой функции

$$
\begin{gathered}
g_{0}^{(s)}(z)=\frac{\sqrt{n-r+1}}{\alpha_{n, r}} \Phi\left(\sqrt{\frac{n-r-m+1}{n-r+1}} \frac{1}{\alpha_{n-r, m}}\right) \alpha_{n, s} z^{n-s}, \\
E_{n-s-1}\left(g_{0}^{(s)}\right)_{2}=\sqrt{\frac{n-r+1}{n-s+1}} \frac{\alpha_{n, s}}{\alpha_{n, r}} \Phi\left(\sqrt{\frac{n-r-m+1}{n-r+1}} \frac{1}{\alpha_{n-r, m}}\right),
\end{gathered}
$$

то, пользуясь последним равенством, запишем оценку снизу

$$
E_{n-s-1}\left(W_{2}^{(r)}\left(\mathscr{K}_{m}, \Phi\right)\right)_{2} \geq E_{n-s-1}\left(g_{0}^{(s)}\right)_{2}=\sqrt{\frac{n-r+1}{n-s+1}} \frac{\alpha_{n, s}}{\alpha_{n, r}} \Phi\left(\sqrt{\frac{n-r-m+1}{n-r+1}} \frac{1}{\alpha_{n-r, m}}\right) .
$$

Сопоставляя оценку сверху (32) с оценкой снизу (33), получаем требуемое равенство (31), чем и завершаем доказательство теоремы 4.

\section{ЛитератУРА}

[1] Смирнов В.И., Лебедев Н.А. Конструктивная теория функиий комплексного переменного (М.-Л.: Наука, 1964).

[2] Абилов В.А., Абилова Ф.В., Керимов М.К. Точнъе оценки скорости сходимости рядов Фуръе функций комплексной переменной в пространстве $L_{2}(D, p(z))$, ЖВММФ 50 (6), 999-1004 (2010).

[3] Шабозов М.Ш., Саидусайнов М.С. Среднеквадратичное приближение функций комплексной переменной рлдами Фуръе в весовом пространстве Бергмана, Владикавк. матем. журн. 20 (1), $86-97$ (2018).

[4] Саидусайнов М.С. Анализ одной теоремы о неравенстве Джексона-Стечкина в пространстве Бергмана $B_{2}$, Тр. ИММ УрО РАН. 24 (4), 217-224 (2018).

[5] Шабозов М.Ш., Саидусайнов М.С. Верхние грани приближения некоторых классов бункиий комплексной переменной рядами Фуръе в пространстве $L_{2}$ и значения $n$-поперечников, Матем. заметки 103 (4), 617-631 (2018).

[6] Бицадзе А.В. Основы теории аналитических функиий комплексного переменного (Наука, М., 1984).

[7] Берг Й., Лефстрем Й. Введение / Интерполяционные пространства (Мир, М., 1980).

[8] Mhaskar N.H. Weighted polynomial Approximation, J. Approx. Theory 46 (1), 100-110 (1986).

[9] Ditzian Z., Totik V. $\mathscr{K}$-functionals and best polynomial approximation in weighted $L^{p}(\mathbb{R})$, J. Approx. Theory 46 (1), 38-41.

[10] Saidusaynov M.S. $\mathscr{K}$-functionals and exact values of $n$-widths in the Bergman space, Ural Math. J. $32(5)$, 74-81.

[11] Шевчук И. А. Приближение многочленами и следы непрерывных на отрезке функций (Наукова думка, Киев, 1992).

\section{Мирганд Шабозович Шабозов}

Таджикский национальный университет,

г. Душанбе, 734025, Республика Таджикистан,

e-mail:shabozov@mail.ru 
Муким Саидусайнович Саидусайнов

Университет Центральной Азии,

г. Душанбе, 734013, Республика Таджикистан,

e-mail: smuqim@gmail.com

M.Sh. Shabozov and M.S. Saidusaynov

Approximation of functions of a complex variable by Fourier sums in orthogonal systems in $L_{2}$

Abstract. The sharp inequalities of Jackson-Stechkin type inequalities between the best approximation $E_{n-s-1}\left(f^{(s)}\right)(s=\overline{0, r}, r \in \mathbb{N})$ of successive derivatives $f^{(s)}(s=\overline{0, r}, r \in \mathbb{N})$ of analytic functions $f \in L_{2}(U)$ in the disk $U:=\{z:|z|<1\}$ as for special module of continuity $\Omega_{m}$ of $m$ th order satisfying the condition

$$
\Omega_{m}\left(f^{(r)}, t\right)_{2} \leq \Phi(t), 0<t<1,
$$

where $\Phi$ is give majorant and also for Peetre $\mathscr{K}$-functional satisfying the constraint

$$
\mathscr{K}_{m}\left(f^{(r)}, t^{m}\right) \leq \Phi\left(t^{m}\right), 0<t<1,
$$

were obtained.

Keywords: the generalized module of continuity, generalized translation operator, orthonormal system of functions, Jackson-Stechkin inequality, $\mathscr{K}$-functional.

Mirgand Shabozovich Shabozov

Tajik National University,

Dushanbe, 734025 Republic of Tajikistan,

e-mail: shabozov@mail.ru

Mukim Saidusaynovich Saidusaynov

University of Central Asia,

Dushanbe, SPCE, 734013 Republic of Tajikistan,

e-mail : smuqim@gmail.com 